\title{
Current Rehabilitation Practices for Children with Cerebral Palsy: Focus and Gaps
}

\author{
Dana Anaby, Nicol Korner-Bitensky, Emma Steven, Stephanie Tremblay, \\ Laurie Snider, Lisa Avery \& Mary Law
}

To cite this article: Dana Anaby, Nicol Korner-Bitensky, Emma Steven, Stephanie Tremblay, Laurie Snider, Lisa Avery \& Mary Law (2017) Current Rehabilitation Practices for Children with Cerebral Palsy: Focus and Gaps, Physical \& Occupational Therapy In Pediatrics, 37:1, 1-15, DOI: 10.3109/01942638.2015.1126880

To link to this article: https://doi.org/10.3109/01942638.2015.1126880

曲 Published online: 11 Feb 2016.

Submit your article to this journal $\pi$

ЦIll Article views: 3464

Q View related articles $₫$

View Crossmark data ¿

Citing articles: 28 View citing articles 5 


\title{
Current Rehabilitation Practices for Children with Cerebral Palsy: Focus and Gaps
}

\author{
Dana Anaby, Nicol Korner-Bitensky, Emma Steven, Stephanie Tremblay, \\ Laurie Snider, Lisa Avery, \& Mary Law
}

\author{
School of Physical and Occupational Therapy, McGill University, Montréal, Québec, \\ Canada
}

\begin{abstract}
Aims: To describe the focus of therapy practices in occupational and physical therapy for school-aged children with cerebral palsy, and better understand whether it is congruent with recommended practices. Methods: A Canada-wide Webbased survey was completed by 62 occupational and 61 physical therapists to identify problems, assessments, and treatment interventions for two case-based scenarios. Data were coded using the International Classification of Functioning, Disability and Health (ICF) definitions for "body functions and structure," "activity and participation," and "environment." Results: Physical therapists, in comparison to occupational therapists, were more likely to select interventions classed in the "body functions and structure" category (34-42\% and 18-20\%, respectively). Both professions focused on "activity and participation" (34-61\%) when identifying problems, assessing, and intervening; attention, however, was mainly directed towards task-oriented activities such as activities of daily living and mobility. Participation in leisure or community-based activities received less attention $(2-15 \%)$. The environment received limited attention for problems and assessments (4-25\%), though it was an important focus of intervention (19-37\%). Conclusions: While body functions and structure are well-addressed, other ICF elements, specifically participation, are poorly integrated into practice. The emerging focus on the environment in therapy intervention, by modifying the context rather than changing aspects of the child, is consistent with current approaches and evidence. Knowledge translation implementation initiatives are recommended to bridge identified gaps.
\end{abstract}

KEYWORDS. Cerebral palsy, focus of intervention, occupational therapy, pediatrics, physical therapy

Conceptual models of disability have undergone a shift in the past two decades, broadening the focus beyond the level of the impairment to account for everyday areas of functioning and role performance. ${ }^{12}$ The International Classification of Functioning, Disability and Health (ICF) is an example of one such model,

Address correspondence to: Dana Anaby, School of Physical and Occupational Therapy, McGill University, Hosmer Room 3023654 promenade Sir-William-Osler, Montréal, QC, Canada H3G 1Y5 (E-mail: dana.anaby@mcgill.ca).

(Received 7 August 2015; accepted 16 November 2015) 
emphasizing the emerging concept of participation as a form of functioning and disability, and placing importance on the role of the environment in determining participation restrictions. ${ }^{28}$ While body functions and structures have been readily addressed in practice for many years, it is now widely recognized that participation is also key to a child's development, health, and well-being, and is one of the most important outcomes of rehabilitation intervention for children with cerebral palsy (CP). ${ }^{18,24}$ The participation of children with physical disabilities, however, is significantly restricted in comparison to their typically developing peers. ${ }^{10,14}$

While knowledge of child participation is increasing, the extent to which it is integrated into practice is unclear. Previous studies that examined therapy practices for children with CP provide preliminary evidence that is, as of yet, inconclusive. Two studies examined the practices of occupational therapists (OTs) and physical therapists (PTs) working with young children ( $<6$ years) with CP. Saleh et al. found that, in Quebec, little emphasis was placed on assessments or interventions specific to leisure, participation, and play. ${ }^{23}$ Overall, clinicians tended to focus more on the ICF's traditional components of "body functions and structure" (e.g., muscle tone, motor control) and "activity" (e.g., gross-motor skills, gait functioning) rather than on "participation" (e.g., socialization, recreation). Similarly, Palisano et al. found that, based on parental reports, interventions generally focused on primary physical impairments. ${ }^{21}$ Another study investigating therapy practices specific to goals and interventions in Alberta revealed that only $15 \%$ of therapists $(n=53)$ identified participation-focused goals, with limited attention to participation as an intervention strategy $(7 \%) .{ }^{9}$ Clearly, a more in-depth, nationwide exploration of therapy practices for school-age children with $\mathrm{CP}$ that is based on clinicians' perspectives is needed.

In conjunction with evolving conceptual models of disability, therapy approaches for children with $\mathrm{CP}$ have been increasingly focused on the child's ability to complete activities and to facilitate their participation in different life settings (i.e., home, school, and community). Concepts emerging from the dynamic systems theory of motor learning as well as ecological approaches have prompted therapists to focus on functional goals within the child's environment. ${ }^{1,26}$ Thus, interventions aim to improve the child's functional activities primarily by changing identified constraints in the task or environment rather than aspects of the child (e.g., body functions and structure) ${ }^{8}$ A randomized controlled trial by Law et al. found that context therapy, which focused on changing the task and/or the environment, was effective in improving the performance of young children with CP. ${ }^{17}$ Another randomized trial demonstrated that therapy focused on changing the performance of a task within the child's natural environment (top-down approach) was more effective than therapy targeting quality of movement (bottom-up, impairment-based approach). ${ }^{13}$ A recent systematic review of the effectiveness of interventions for children with CP revealed that the majority of studies focused on outcomes at the level of the activity and their intervention approaches were found most effective ("green go"). ${ }^{20}$ Examples of these approaches included context-therapy, constraint-induced movement therapy (CIMT), and goal-directed therapy, among others. Clearly, evidence for contemporary interventions is available supporting a shift towards more ecological, activity-based approaches. ${ }^{16}$ 
The purpose of this study was to describe current therapy practices applied by OTs and PTs in the management of school-age children with CP. Specifically, the research question was: How does each profession allocate focus across the key elements of the ICF (i.e., "body functions and structure," "activity and participation," and "environment") when identifying problems, selecting assessments, and proposing treatment interventions? The survey also explores the use of standardized assessments and protocols of intervention (e.g., CIMT). Such information will help better understand whether current practices are in line with up-to-date approaches and recommended practice, and identify specific knowledge-to-practice gaps that can then be addressed.

\section{METHODS}

This cross-sectional study was approved by the Institutional Review Board of McGill University. Eligible participants were registered OTs and PTs in Canada who have provided services for at least 4 months in the past year for school-age children (aged 5-10 years) with CP. The Canadian Association of Occupational Therapists (CAOT) and the Canadian Physiotherapy Association (CPA) Web sites provided the names and contact information of participating provincial and territorial licensing agencies or recognized professional associations of OTs and PTs in Canada. All possible agencies were approached, with nine of the 13 physical therapy organizations agreeing to participate as well as 10 out of the 12 provincial occupational therapy organizations (response rate between $69 \%$ and $83 \%$ ), forwarding the online survey to their members. Additionally, all members with electronic contact information listed on the CAOT Web site were forwarded the survey and encouraged to in turn forward it to colleagues who would potentially meet the eligibility criteria, therefore promoting a snowball sampling effect. Pediatric clinicians who consented online and were determined to be eligible based on the above criteria responded to questions on the actual management of children with $\mathrm{CP}$ using case scenarios describing two different children with CP.

\section{Participants}

A total of 366 clinicians responded to the online invitation to complete a survey, of which 281 were eligible responders and 123 participants completed the survey ( $44 \%$ response rate). The sample involved both OTs and PTs from across Canada working in a variety of settings including schools $(41 \%)$, outpatient clinics $(38 \%)$, community organizations (7\%), and hospitals (6\%), among others (see Table 1). Sixty-seven percent worked full-time, and the majority of responders $(54 \%)$ met with two to five children with $\mathrm{CP}$ every week. Certain responders were eligible but did not complete the survey $(n=158)$, and chi-square tests indicated that this group was not significantly different from those that fully completed it $(n=123)$ in terms of available demographic characteristics (e.g., language, province), therefore attenuating the potential risk of sample bias.

\section{Survey}

FluidSurveys. The online survey was created using FluidSurveys, a Web-based software specializing in collecting responses using questionnaires and forms. ${ }^{11}$ The 
TABLE 1. Sample Characteristics

\begin{tabular}{|c|c|c|c|}
\hline & & $N$ & Percent \\
\hline \multirow[t]{2}{*}{ Profession } & OT & 62 & 50.4 \\
\hline & PT & 61 & 49.6 \\
\hline \multirow[t]{2}{*}{ Sex } & Male & 6 & 4.9 \\
\hline & Female & 117 & 95.1 \\
\hline \multirow[t]{2}{*}{ Education } & Bachelor & 88 & 71.5 \\
\hline & Masters & 34 & 27.6 \\
\hline \multirow{4}{*}{$\begin{array}{c}\text { Years of experience } \\
\text { with CP patients }\end{array}$} & $<2$ & 12 & 9.8 \\
\hline & $2-5$ & 33 & 26.8 \\
\hline & $6-10$ & 17 & 13.8 \\
\hline & $>10$ & 61 & 49.6 \\
\hline \multirow[t]{10}{*}{ Province } & Alberta & 15 & 12.2 \\
\hline & British Columbia & 15 & 12.2 \\
\hline & Manitoba & 9 & 7.3 \\
\hline & New Brunswick & 13 & 10.6 \\
\hline & Newfoundland and Labrador & 3 & 2.4 \\
\hline & Nova Scotia & 3 & 2.5 \\
\hline & Ontario & 28 & 22.8 \\
\hline & Quebec & 26 & 21.1 \\
\hline & Saskatchewan & 10 & 8.1 \\
\hline & Yukon & 1 & 0.8 \\
\hline \multirow[t]{3}{*}{ Environment } & Urban & 81 & 65.9 \\
\hline & Suburban & 16 & 13.0 \\
\hline & Rural & 26 & 21.1 \\
\hline
\end{tabular}

survey was available to participants in both English and French, and was designed for the accessibility to the greatest number of responders as well as to be of a reasonable length to optimize response time. Case scenarios were developed, pilottested, and validated; expert clinicians and researchers serving children with $\mathrm{CP}$ were asked to create a "typical" child and describe them in terms of gross- and finemotor functions, sensory function, behavior, language, psycho-educational and cognitive functions, activities related to participation, family structure, and physical and social environments, representing a range of key elements of the ICF. ${ }^{23}$ The first case described a 6-year-old girl with hemiplegia and the second case illustrated a 7-year-old boy with diplegia; both were included in regular schools.

A standardized and validated questionnaire tested among pediatric clinicians was used, with slight adjustments to reduce the length of administration. ${ }^{23}$ The questionnaire consists of four components: (1) clinician characteristics; (2) work setting; (3) the case vignette(s); and (4) open-ended questions related to the vignette including identification of problems, and choices of assessments and interventions that would typically be used in clinical practice for the child described, as well as specific referrals to other services. For each case, clinicians were prompted to answer open-ended questions based on actual daily practice. Specifically, they were asked to identify problems and suggest assessments and intervention approaches they would use. The therapists could list up to 10 answers for each section. A certificate of completion recognizing their contribution to the study was printable upon completing the survey. 


\section{Data Analysis}

Each completed questionnaire was reviewed by two independent evaluators, and the data regarding problems, assessments, and interventions were coded according to a coding system discussed and developed by the authors that incorporated the ICF linking rules proposed by Ceiza et al. ${ }^{7}$ The codes were reviewed by another member of the research team and, where appropriate, grouped to eliminate redundancy. In addition, a third person, an investigator on the project, verified the codes when the terms were ambiguous. Finally, codes were grouped according to the ICF classification: body functions and structure, activity and participation, and environmental factors. Specific subthemes for each category of the ICF were developed while drawing on the Canadian Model of Occupational Performance components and terminology. 6

As suggested by Whiteneck and Dijkers, a conceptual distinction between "activity" and "participation" was made, where activity occurs at the personal level (e.g., getting dressed) and participation involves role performance at a societal level (e.g., joining a team sport). ${ }^{27}$ An initial coding structure (including subthemes and examples) was developed and reviewed by three independent OTs with expertise in pediatrics, and feedback was provided. Examples of categories and subthemes are described in Table 2.

To describe the distribution of focus areas by the ICF categories, frequency scores were calculated for each of the subcategories. These scores represent the proportion of category responses with equal weight given to each therapist. To examine the differences in rates of use of ICF categories/subcategories between the professions and across cases, Fisher's exact tests were used. To describe and compare the rates of use of standardized assessments between professions, the $z$ statistic was used. The assessments were included in the analysis if they were cited by at least $5 \%$ of therapists. In addition, the number of times each standardized assessment was reported by therapists was counted in proportion to overall cited assessments and ranked to exemplify the most common assessments used in practice. Finally, a total count of therapists who suggested specific therapeutic approaches was tallied/calculated across cases and professions.

\section{RESULTS}

\section{Overall Patterns of Practice}

OTs and PTs focused considerably on the "activity and participation" category (34-61\%) when identifying problems, assessing and intervening across both cases. Attention, however, was mainly directed towards task-oriented activities (19-56\%) such as activities of daily living (ADLs; e.g., dressing), mobility (e.g., gross-motor skills), and academics (e.g., writing), whereas participation, particularly in leisure or community-based activities (e.g., biking, hockey), received the least attention $(2-15 \%)$.

\section{Focus of Occupational Therapy}

As shown in Figure 1, OTs mostly identified, in both cases, activity-based problems related to mobility/motor skills (15-25\%), ADLs (13-18\%), and body functions of 
TABLE 2. Examples of Categories and Subthemes Used for Coding Data

\begin{tabular}{|c|c|c|c|c|}
\hline $\begin{array}{l}\text { ICF } \\
\text { Categories }\end{array}$ & Subthemes & Problems & Assessments & Interventions \\
\hline \multirow[t]{3}{*}{$\begin{array}{l}\text { Body } \\
\text { functions and } \\
\text { structure }\end{array}$} & $\begin{array}{l}\text { B1. Muscle/Joint } \\
\text { mobility/Motor } \\
\text { control }\end{array}$ & $\begin{array}{l}\text { Lower extremity } \\
\text { spasticity }\end{array}$ & $\begin{array}{l}\text { Ashworth Scale; } \\
\text { BOT-2 }^{\mathrm{a}}\end{array}$ & $\begin{array}{l}\text { NDT }^{\mathrm{b}} \text { Strengthening } \\
\text { exercises }\end{array}$ \\
\hline & $\begin{array}{l}\text { B2. En- } \\
\text { durance/Balance }\end{array}$ & Poor endurance & Berg Balance Scale & Cardio training \\
\hline & $\begin{array}{l}\text { B3. Cogni- } \\
\text { tive/Emotional }\end{array}$ & $\begin{array}{l}\text { Spatial/Visual } \\
\text { perception; } \\
\text { Attention }\end{array}$ & Beery VMIc/DVPT-2 ${ }^{d}$ & $\begin{array}{l}\text { Visual perception } \\
\text { interventions }\end{array}$ \\
\hline \multirow[t]{4}{*}{ Activity } & $\begin{array}{l}\text { D1. Moving } \\
\text { around/Mobility }\end{array}$ & $\begin{array}{l}\text { Stair climbing; } \\
\text { Positioning }\end{array}$ & $\begin{array}{l}\text { GMFM }^{e}-66 \text { or }-88 ; \\
\text { MABC }^{f} 2^{f}\end{array}$ & $\begin{array}{l}\text { Gross/fine motor } \\
\text { skills }\end{array}$ \\
\hline & D2. $A D L s^{g}$ & $\begin{array}{l}\text { Toileting; } \\
\text { Independence } \\
\text { with dressing }\end{array}$ & PEDI ${ }^{\mathrm{h}}$ & $\begin{array}{l}\text { Functional tasks } \\
\text { with adaptations }\end{array}$ \\
\hline & D3. Academic & $\begin{array}{l}\text { Math; Written } \\
\text { productivity }\end{array}$ & $\begin{array}{l}\text { McMaster printing } \\
\text { assessment tool }\end{array}$ & $\begin{array}{l}\text { Handwriting } \\
\text { program } \\
\text { (ABCBoom, } \\
\text { Handwriting } \\
\text { Without Tears) }\end{array}$ \\
\hline & D4. Communication & Social skills & Verbal interactions & $\begin{array}{l}\text { Computer/iPad for } \\
\text { communication }\end{array}$ \\
\hline \multirow[t]{2}{*}{ Participation } & $\begin{array}{l}\text { D5. General } \\
\text { participation }\end{array}$ & Riding bike; Sports & $\begin{array}{l}\text { COPM }^{\mathrm{i}} \text {; Narrative } \\
\text { participation }\end{array}$ & $\begin{array}{c}\text { Adapt games/sports } \\
\text { (adapted bike, } \\
\text { sledge hockey) }\end{array}$ \\
\hline & $\begin{array}{l}\text { D6. School } \\
\text { participation }\end{array}$ & Gym participation & $\begin{array}{l}\text { SFA; } \text { Physical } \\
\text { education } \\
\text { observation; } \\
\text { Observation at re- } \\
\text { cess/playground }\end{array}$ & Gym participation \\
\hline \multirow[t]{3}{*}{ Environment } & E1. Physical & $\begin{array}{l}\text { Home access; } \\
\text { Transportation }\end{array}$ & $\begin{array}{l}\text { Home accessibility } \\
\text { assessment }\end{array}$ & Supportive seating \\
\hline & E2. Social/Cultural & Family supports & Interview with family & $\begin{array}{l}\text { Educate school on } \\
\text { CP and its effects; } \\
\text { Consult with gym } \\
\text { teacher to } \\
\text { promote inclusion }\end{array}$ \\
\hline & E3. Institutional & $\begin{array}{l}\text { Lack of support staff } \\
\text { at school }\end{array}$ & & $\begin{array}{l}\text { Adapt school } \\
\text { curriculum and } \\
\text { program; Explore } \\
\text { funding options } \\
\text { for renovations to } \\
\text { environment; Ask } \\
\text { for one-on-one } \\
\text { support at school }\end{array}$ \\
\hline Other & $\begin{array}{l}\text { O1. Medical history } \\
\text { O2. Observation } \\
\text { O3. Multidisciplinary }\end{array}$ & & Chart review & \\
\hline
\end{tabular}

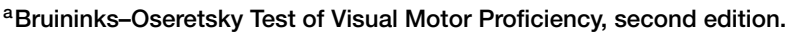

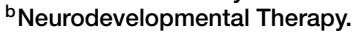

${ }^{\mathrm{c} B e e r y-B u k t e n i c a}$ Developmental Test of Visual Motor Integration.

${ }^{\mathrm{d}}$ Developmental Test of Visual Perception, second edition.

${ }^{\mathrm{e}}$ Gross Motor Function Measure.

${ }^{f}$ Movement Assessment Battery for Children, second edition.

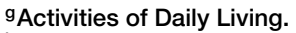

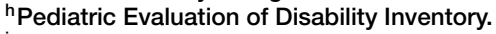

'Canadian Occupational Performance Measure.

iSchool Function Assessment. 
motor control (13-15\%), whereas participation-based issues received the least attention (6-8\%). Considerable focus was directed to identifying barriers within the physical environment (15\%), in particular in Case 2. These domains were also targeted when selecting assessments. OTs, in Case 1, focused on evaluating aspects of the child (muscle/joint mobility and cognitive abilities; $17 \%$ and $10 \%$, respectively) followed by activity performance (ADLs and academic activities, $13 \%$ and $10 \%$, respectively) and the physical environment (10\%). Notably, OTs tended to report a generic nonspecific assessment of "observation" quite often (15\% in Case 2; $10 \%$ in Case 1) and this was classified as "other." Therapy intervention was most often activity-focused (ADLs; $25 \%$ ) and attention was equally directed towards modifying the environment (physical; 17\%) and the child's body functions (muscle/joint mobility; $17.5 \%$ ).

In Case 2, OTs' assessments focused on the physical environment (e.g., home accessibility, equipment related to mobility; $22 \%$ ) followed by body functions (muscle/joint mobility; 17\%) and ADLs (11\%), and these domains also received attention when providing interventions. Indeed, OTs focused mostly on modifying the physical environment (26\%), followed by task-oriented activities (ADLs; $18 \%$ using strategies for task accomplishment or compensatory approaches) and improving body functions related to muscle/joint mobility (18\%) - the latter illustrating impairment-level approaches where the focus is on changing the child. In both cases, little attention was directed towards assessing and intervening at the level of participation (e.g., recreational out-of-school activities; 1.6-7.6\%).

Similar attention was given in Case 1 to "activity and participation" across all elements of practice: problems, assessments, and interventions. However, while similar attention is directed to both identifying and assessing problems related to "body functions and structure" $(28 \%)$ as well as to the "environment" $(16 \%)$, OT interventions (in Case 1) are more focused on changing the environment (30\%) rather than body functions (20\%). Relatively similar patterns were observed when analyzing Case 2, where OTs assessed aspects of body functions and environment equally ( $23 \%$ and $25 \%$, respectively) but intervened more at the level of the environment $(37 \%)$.

\section{Focus of Physical Therapy}

As shown in Figure 2, PTs tended to identify problems related to "body functions" (e.g., muscle/joint mobility) and "activity" (mobility/motor skills). In both cases, PTs focused mainly on assessing body functions related to muscle/joint mobility $(37-42 \%)$ and activities related to mobility and motor skills (35-37\%). While in both cases PTs targeted mostly muscle/joint mobility (in the body functions and structure category, addressing the impairments using, for instance, stretching exercises; 30-33\%) when providing interventions, however there were some differences across cases. In Case 1, attention was designated towards participation $(12 \%)$ and in Case 2, the physical environment received attention (23\%). PTs directed little attention towards assessing the environment (3.7\% in Case $1 ; 9.1 \%$ in Case 2$)$; however, when intervening they tended to focus on it more $(19 \%$ in Case $1 ; 28 \%$ in Case 2). 

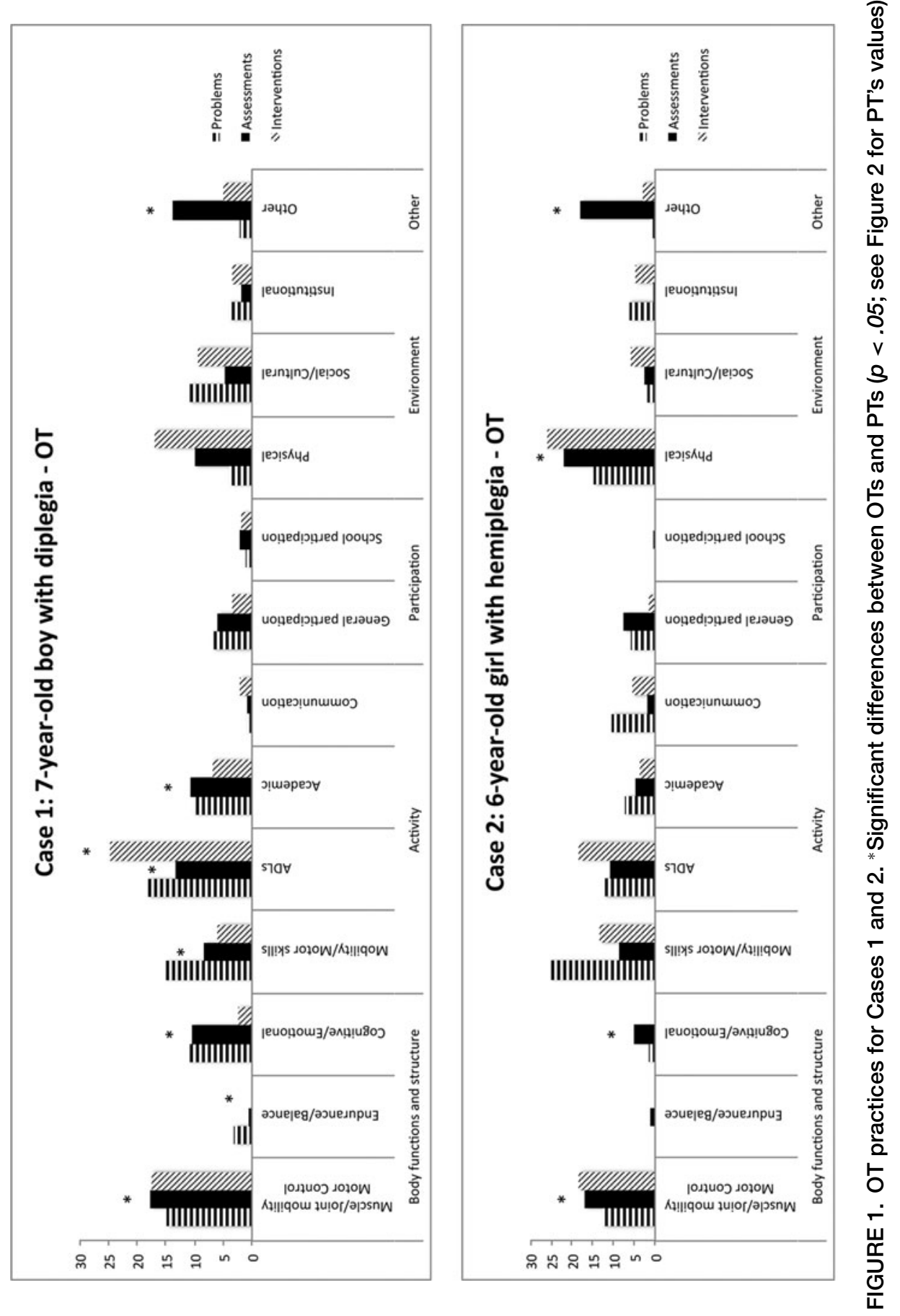


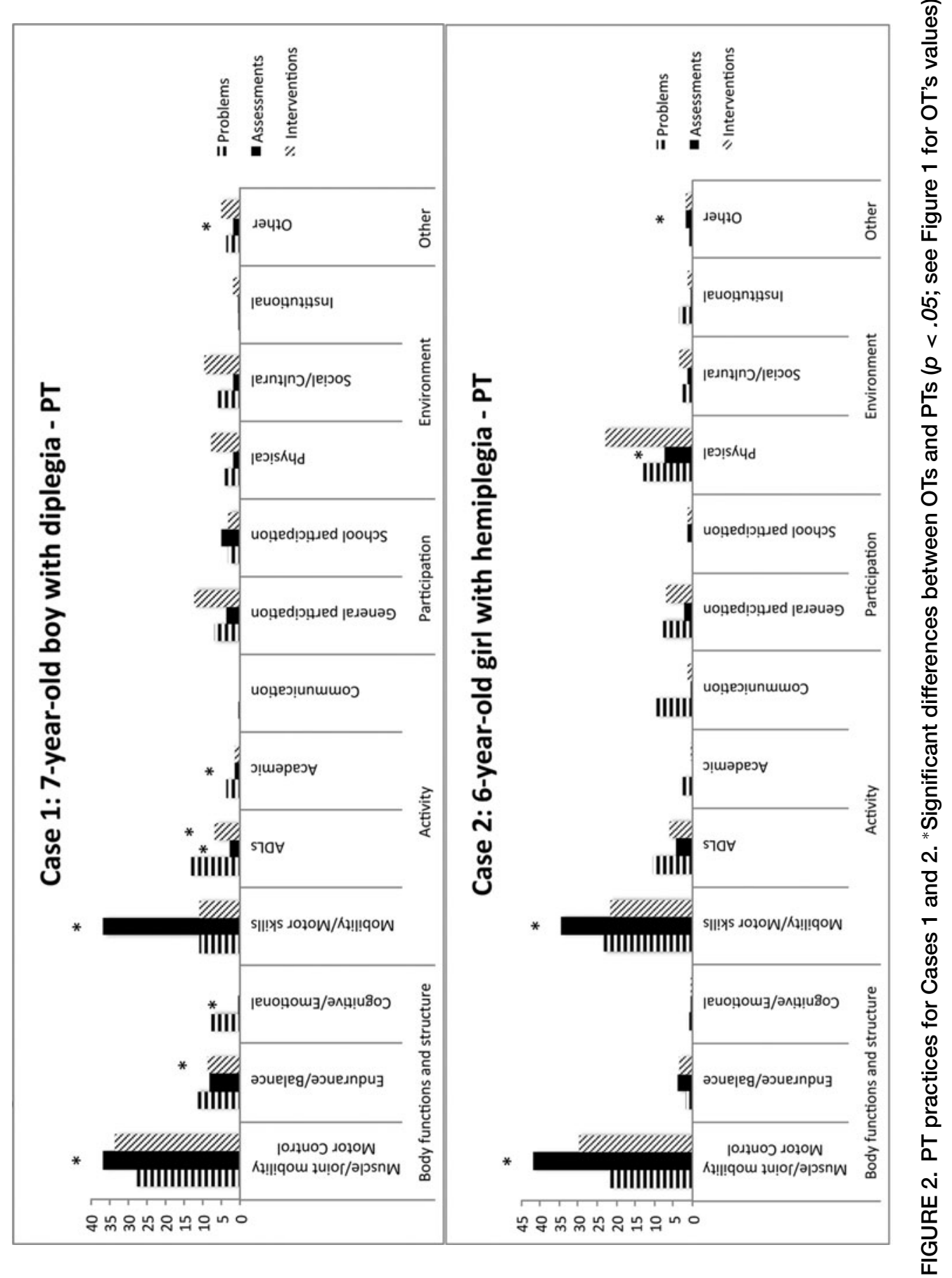




\section{Differences Between Focus of OT and PT Interventions}

There were no significant differences between OTs and PTs with respect to their classification of problems across both cases $(z=0.22, p=.26$ for Case $1 ; z=0.5$, $p=.73$ for Case 2). There was, however, a difference in the allocation of assessment tools between OTs and PTs. PTs were more likely to choose assessments in the "mobility/motor skills" category $(z=-3.54, p<.001$ for Case $1 ; z=-2.95$, $p=.003$ for Case 2) and less likely to select assessments in the "environment" category $(z=2.22, p=.03$ for Case $1 ; z=2.04, p=.04$ for Case 2). For the case involving a child with diplegia (Case 1), the PTs were also significantly less likely to select an assessment in the "ADLs" and "academic" categories $(z=2.05, p=$ $.04 ; z=2.11, p=.035$, respectively). A similar trend was observed for the case involving hemiplegia (Case 2) but was not statistically significant $(z=1.22, p=$ .22). Additionally, there was a significant difference in the categories chosen for interventions between the two groups for Case 1, with PTs being more likely to select interventions classed in the "body functions and structure" category $(z=$ $-2.5, p=.01)$, and less likely to select "ADL" interventions $(z=2.66, p=.008)$.

\section{Most Commonly Used Standardized Outcome Measures}

While PTs reported using standardized assessments more often (56\% for Case 1 ; $39 \%$ for Case 2) than OTs ( $44 \%$ for Case $1 ; 27 \%$ for Case 2$)$, these differences were not statistically significant for either Case $1(z=-1.24, p=.22)$ or Case 2 $(z=-1.22, p=.22)$. A wide variety of assessments were listed (38 standardized assessments total). Table 3 lists the five most common measures used by each profession. Overall, across all therapists, range of motion (ROM) and goniometry (body functions and structure) were most often cited (45\%), followed by the Gross Motor Function Measure (GMFM; activity, 30\%) and the Beery-Buktenica Developmental Test of Visual Motor Integration (body functions and structure, 26\%). The Canadian Occupational Performance Measure (COPM), which assesses performance in different participation domains, was ranked fifth overall $(18 \%)$, specifically third among OTs (26\%), and 11th among PTs (9\%). Measures that explicitly assess participation were rarely cited (e.g., LIFE-H, 2.6\%; PACS-Pediatrics Activity Card Sort, 1.8\%) and standardized assessments of the environment were not reported at all.

As shown in Table 3, the five most common outcome measures listed by PTs are for body functions and structure, with the exception of the GMFM, which assesses activity. OTs also used assessments related to body functions and structure and activity (mobility/motor skills) and included aspects of participation (measured by the COPM) in their evaluation process.

\section{Use of Clinical Protocols for Interventions}

A relatively low percentage of therapists suggested the use of neurodevelopmental therapy (NDT). This was observed for each of the cases and for each profession (OTs, 18\%; PTs, 13\%). Evidence-based protocols such as CIMT were rarely cited (less than $1 \%$ of responses) whereas context-therapy and cognitive orientation to daily occupational performance (CO-OP) were not explicitly reported across cases and professions. 
TABLE 3. Most Common Standardized Assessments Used by Clinicians

\begin{tabular}{|c|c|c|}
\hline & Assessment & Theme \\
\hline \multicolumn{3}{|c|}{ Occupational therapists } \\
\hline 1 & Beery $\mathrm{VMI}^{\mathrm{a}}$ & Cognitive ${ }^{b}$ \\
\hline 2 & ROMc/Goniometry (30\%) & Muscle/Joint mobility/Motor control ${ }^{b}$ \\
\hline 3 & $\operatorname{COPM}^{d}(26 \%)$ & General participation ${ }^{e}$ \\
\hline 4 & BOT-2 ${ }^{f}(21 \%)$ & Muscle/Joint mobility/Motor control ${ }^{b}$ \\
\hline 5 & QUEST $^{\mathrm{g}}(11 \%)$ & Mobility/Motor skills ${ }^{h}$ \\
\hline \multicolumn{3}{|c|}{ Physical therapists } \\
\hline 1 & ROM/Goniometry (60\%) & Muscle/Joint mobility/Motor control \\
\hline 2 & GMFM $^{i}(58 \%)$ & Mobility/Motor skills ${ }^{h}$ \\
\hline 3 & Manual Muscle Testing (21\%) & Muscle/Joint mobility/Motor control \\
\hline 4 & Berg Balance Scale (19\%) & Endurance/Balance ${ }^{\mathrm{b}}$ \\
\hline 5 & BOT-2 (19\%) & Muscle/Joint mobility/Motor control \\
\hline \multicolumn{3}{|c|}{ All clinicians } \\
\hline 1 & ROM/Goniometry (45\%) & Muscle/Joint mobility/Motor controlb \\
\hline 2 & GMFM $(30 \%)$ & Mobility/Motor skills ${ }^{\mathrm{b}}$ \\
\hline 3 & Beery VMI (26\%) & Cognitive $^{\mathrm{b}}$ \\
\hline 4 & BOT-2 (20\%) & Muscle/Joint mobility/Motor control \\
\hline 5 & COPM (18\%) & General participation ${ }^{e}$ \\
\hline
\end{tabular}

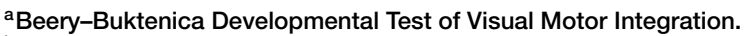

${ }^{b}$ Body functions and structures.

${ }^{\mathrm{c}}$ Range of Motion.

dCanadian Occupational Performance Measure.

eParticipation.

fBruininks-Oseretsky Test of Motor Proficiency, second edition.

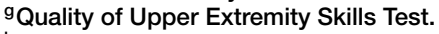

${ }^{\text {h}}$ Activity.

'Gross Motor Function Measure.

\section{DISCUSSION}

This study sought to examine the current focus of rehabilitation practices when providing services to school-age children with CP. Findings reveal some gaps between what we know and what we do in practice. To illustrate, considerable attention is directed towards the ICF category of body functions and structure involving a focus on quality and patterns of movement (muscle tone, ROM, motor control), when assessing and intervening, in particular among PTs. Such "bottom up," impairment-level approaches are not grounded in evidence and it is uncertain whether such efforts will translate into improved functional independence and increased participation in different life situations. ${ }^{16,19}$

At the same time, the focus on activity and participation is mainly directed to task-oriented activities such as ADLs and mobility/motor skills, while limited focus is directed to participation in community-based leisure activities (such as hockey and bike riding). Previous research examining therapy practices indicates similar patterns (among younger children), where interventions focus on primary impairment or body functions and motor skills. ${ }^{23}$ Although research indicates that practitioners including therapists, pediatric physicians, and neurologists value the outcome of social participation and consider it as a relevant area of functioning, and $60 \%$ of our sample indicated that a change in their clinical practice has occurred in the last year, gaps still exist and the shift has not been fully incorporated. . $^{3,24}$

The observed focus on the environment as a target of intervention is encouraging as it is in line with contemporary therapy approaches as well as evidence related to 
context therapy whose focus is on modifying the task and/or the environment. ${ }^{16,17}$ While the physical environment received most of the attention, we recommend consideration of the social and attitudinal environment, such as lack of social support as well as lack of knowledge, and at times willingness of community service providers and staff to accommodate their programs that often restrict participation of children with disabilities. ${ }^{2}$ A recently developed environmentbased intervention called Pathways and Resources for Engagement and Participation (PREP), which aims to remove barriers within the environment and coach families, was found to be effective in promoting youth's participation in community-based leisure activities. ${ }^{4,5,15}$ In this approach, all elements of the environment are addressed, including social, cultural, attitudinal, and institutional, and intervention occurs in the community where activities take place.

Other specific approaches that have established evidence of effectiveness ("green light" interventions) such as CIMT were scarcely mentioned by study participants. NDT, classified by Novak and colleagues (2013) as an ineffective "red light" intervention in which, for the most part, evidence base is unfavorable, was reported in some of the cases (range 13-18\%) as a clinical protocol used in practice; however, percentages were relatively low and showed a decline in comparison to previous research (where the prevalence was about $30 \%$ ). ${ }^{20,23}$

A "know-do" gap was also observed for therapists' use of standardized assessments. While the COPM, a measure that assesses activity performance and participation, received a certain degree of attention in practice (19\%), the most commonly used assessments still fall within the "body functions and structure" category (or impairment-level assessments such as the Beery-VMI and goniometry). Assessments that lie within the "activity" category were mainly directed to motor skills and mobility (e.g., GMFM), whereas psychometrically sound assessments of participation and the environment such as the Children's Assessment of Participation and Enjoyment (CAPE), the Participation and Environment Measure for Children and Youth (PEM-CY), and the Craig Hospital Inventory of Environmental Factors (CHIEF) were rarely reported. Similar patterns were also evident in a study exploring the use of assessments by Thai rehabilitation specialists across the ICF categories, suggesting a widespread gap. ${ }^{25}$

\section{Limitations and Further Directions}

As the survey is a self-reported consideration of what clinicians would do in practice, it represents what they would intend to do but may not, in fact, reflect the clinical realities of their practice (secondary to time management difficulties, work environment culture/pressures, limited standardized assessments available). Future studies can include chart reviews to record what is actually being done in therapy, followed by an interview with therapists to discuss their practice behaviors including potential gaps between desired and actual practice.

Another limitation is that the case scenarios represent a narrow age group of children with CP (ages 6 and 7 years); however, this represents a critical time point in terms of transitioning to school, as well as falling within a developmental stage where improvement in motor growth curves is no longer observed and, consequently, implies using more adaptive rather than remedial impairment-based approaches. ${ }^{22}$ Nevertheless, it would be important to widen the targeted age range 
and verify whether this has any impact on practice. Finally, while all Canadian agencies and associations of OT and PT were approached, resulting in representatives from the majority of provinces (see Table 1), caution should be taken when generalizing the findings.

An a priori power analysis was not conducted because expected differences among groups were difficult to estimate. However, the differences between professions (with respect to how the scenarios were classified) were statistically significant based on both the traditional chi-square and the Fisher's exact tests; this indicates an adequate power for comparisons.

\section{Conclusions and Implications}

Some gaps between current and recommended practice exist across OT and PT practice, illustrating the need to expand the scope of practice to include participation-related domains beyond ADLs and mobility. Considerable attention is still directed to changing aspects of the child (i.e., body functions and structure) although evidence for its benefits is limited. Current approaches encourage a more activity-based ecological intervention focus. Furthermore, while the emerging focus on the environment in therapy intervention (which modifies the context rather than the child) is encouraging, it is largely limited to the physical environment. Finally, knowledge translation implementation strategies (e.g., sharing educational material through a Web site/workshop, facilitating case-based tutorials/learning sessions) are therefore needed to bring about a change in practice towards a greater emphasis on all aspects of participation and the environment.

Declaration of Interest: The authors report no conflict of interest. The authors alone are responsible for the content and writing of this article.

\section{FUNDING}

The authors acknowledge funding from the Edith Strauss Foundation (G235149 Richard \& Edith Strauss X-C 218563).

\section{ABOUT THE AUTHORS}

Dana Anaby and Nicol Korner-Bitensky are affiliated with the School of Physical and Occupational Therapy, McGill University, Quebec, Canada. Emma Steven is an occupational therapist at the Centre de réadaptation MAB-Mackay du CIUSSS du Centre-Ouest-de-l'̂̂le-de-Montréal, Quebec, Canada. Stephanie Tremblay and Laurie Snider are also affiliated with the School of Physical and Occupational Therapy, McGill University, Quebec, Canada. Lisa Avery is from Avery Information Services Ltd., and Mary Law is from CanChild, McMaster University, Ontario, Canada.

\section{REFERENCES}

[1] Ahl, L. E., Johansson, E., Granat, T., \& Carlberg, E. B. (2005). Functional therapy for children with cerebral palsy: An ecological approach. Developmental Medicine and Child Neurology, 47, 613-619. 
[2] Anaby, D., Hand, C., Bradley, L., Direzze, B., Forhan, M., Digiacomo, A., \& Law, M. (2013). The effect of the environment on participation of children and youth with disabilities: A scoping review. Disability and Rehabilitation, 35, 1589-1598. doi: 10.3109/09638288.2012.748840

[3] Anaby, D., Korner-Bitensky, N., Law, M., \& Cormier, I. (2015). Focus on participation for children and youth with disabilities: Supporting therapy practice through a guided knowledge translation process. British Journal of Occupational Therapy, 78, 440-449. doi: 10.1177/0308022614563942

[4] Anaby, D. R., Law, M. C., Majnemer, A., \& Feldman, D. (2015). Opening doors to participation of youth with physical disabilities: An intervention study [Favoriser la participation des adolescents ayant des handicaps physiques: Étude d'intervention]. Canadian Journal of Occupational Therapy. doi: 10.1177/0008417415608653

[5] Anaby, D., Law, M., Teplicky, R., \& Turner, L. (2015). Focusing on the environment to improve youth participation: Experiences and perspectives of occupational therapists. International Journal of Environmental Research and Public Health, 12, 13388-13398. doi: 10.3390/ijerph121013388

[6] CAOT. (2002). Enabling occupation: An occupational therapy perspective. Ottawa, ON: CAOT Publications ACE.

[7] Cieza, A., Geyh, S., Chatterji, S., Kostanjsek, N., Üstün, B., \& Stucki, G. (2005). ICF linking rules: An update based on lessons learned. Journal of Rehabilitation Medicine, 37, 212-218. doi: 10.1080/16501970510040263

[8] Darrah, J., Law, M. C., Pollock, N., Wilson, B., Russell, D. J., Walter, S. D., ... Galuppi, B. (2011). Context therapy: A new intervention approach for children with cerebral palsy. Developmental Medicine and Child Neurology, 53, 615-620. doi: 10.1111/j.14698749.2011.03959.x

[9] Darrah, J., Wiart, L., \& Magill-Evans, J. (2008). Do therapists' goals and interventions for children with cerebral palsy reflect principles in contemporary literature? Pediatric Physical Therapy, 20, 334-339.

[10] Engel-Yeger, B., Jarus, T., Anaby, D., \& Law, M. (2009). Differences in patterns of participation between youths with cerebral palsy and typically developing peers. American Journal of Occupational Therapy, 63, 96-104.

[11] FluidSurveys. (2015). FluidSurveys: Online survey software. Retrieved from http://fluidsurveys.com/

[12] Hemmingsson, H., \& Jonsson, H. (2005). An occupational perspective on the concept of participation in the International Classification of Functioning, Disability and Health: Some critical remarks. American Journal of Occupational Therapy, 59, 569-576.

[13] Ketelaar, M., Vermeer, A., Hart, H. t., van Petegem-van Beek, E., \& Helders, P. J. (2001). Effects of a functional therapy program on motor abilities of children with cerebral palsy. Physical Therapy, 81, 1534-1545.

[14] King, G., Law, M., Hurley, P., Petrenchik, T., \& Schwellnus, H. (2010). A developmental comparison of the out-of-school recreation and leisure activity participation of boys and girls with and without physical disabilities. International Journal of Disability Development and Education, 57, 77-107.

[15] Law, M., Anaby, D., Imms, C., Teplicky, R., \& Turner, L. (2015). Improving the participation of youth with physical disabilities in community activities: An interrupted time series design. Australian Occupational Therapy Journal, 62, 105-115. doi: 10.1111/1440-1630.12177

[16] Law, M., \& Darrah, J. (2014). Emerging therapy approaches: An emphasis on function. Journal of Child Neurology, 29, 1101-1107. doi: 10.1177/0883073814533151

[17] Law, M., Darrah, J., Pollock, N., Wilson, B., Russell, D. J., Walter, S. D., ... Galuppi, B. (2011). Focus on function: A cluster, randomized controlled trial comparing child- versus context-focused intervention for young children with cerebral palsy. Developmental Medicine and Child Neurology, 53, 621-629. doi: 10.1111/j.1469-8749.2011.03962.x

[18] McIntyre, S., Novak, I., \& Cusick, A. (2010). Consensus research priorities for cerebral palsy: A Delphi survey of consumers, researchers, and clinicians. Developmental Medicine and Child Neurology, 52, 270-275. 
[19] Novak, I. (2012). Evidence to practice commentary: Is more therapy better? Physical and Occupational Therapy in Pediatrics, 32, 383.

[20] Novak, I., Mcintyre, S., Morgan, C., Campbell, L., Dark, L., Morton, N., ... Goldsmith, S. (2013). A systematic review of interventions for children with cerebral palsy: State of the evidence. Developmental Medicine and Child Neurology, 55, 885-910.

[21] Palisano, R. J., Begnoche, D. M., Chiarello, L. A., Bartlett, D. J., McCoy, S. W., \& Chang, H.-J. (2012). Amount and focus of physical therapy and occupational therapy for young children with cerebral palsy. Physical \& Occupational Therapy in Pediatrics, 32, 368-382. doi: 10.3109/01942638.2012.715620

[22] Rosenbaum, P. L., Walter, S. D., Hanna, S. E., Palisano, R. J., Russell, D. J., Raina, P., ... Galuppi, B. E. (2002). Prognosis for gross motor function in cerebral palsy: Creation of motor development curves. JAMA, 288, 1357-1363.

[23] Saleh, M., Korner-Bitensky, N., Snider, L., Malouin, F., Mazer, B., Kennedy, E., \& Roy, M. A. (2008). Actual vs. best practices for young children with cerebral palsy: A survey of paediatric occupational therapists and physical therapists in Quebec, Canada. Developmental Neurorehabilitation, 11, 60-80.

[24] Schiariti, V., Mâsse, L. C., Cieza, A., Klassen, A. F., Sauve, K., Armstrong, R., \& O’Donnell, M. (2014). Toward the development of the International Classification of Functioning Core Sets for children with cerebral palsy: A global expert survey. Journal of Child Neurology, $29,582-591$.

[25] Tantilipikorn, P., Watter, P., \& Prasertsukdee, S. (2012). Identifying assessment measures and interventions reported for Thai children with cerebral palsy using the ICF-CY framework. Disability and Rehabilitation, 34, 1178-1185.

[26] Thelen, E. (1987). Motor development and self-regulation in the early years. In E. Nancy (Ed.), Contemporary topics in developmental psychology (pp. 3-33). New York: Wiley.

[27] Whiteneck, G., \& Dijkers, M. P. (2009). Difficult to measure constructs: Conceptual and methodological issues concerning participation and environmental factors. Archives of Physical Medicine and Rehabilitation, 90, S22-35.

[28] WHO. (2001). International Classification of Functioning, Disability and Health. Geneva: WHO. 